\title{
Dynamic failure mechanism of copper foil in laser dynamic flexible forming
}

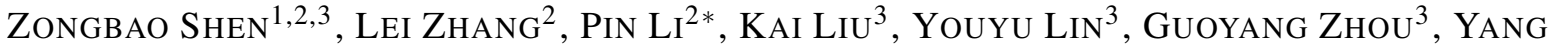 \\ WANG $^{3}$, JiNDIAN ZHANG ${ }^{2}$, HUIXIA LIU ${ }^{2}$, XIAO WANG ${ }^{2}$ \\ ${ }^{1}$ School of Materials Science and Engineering, Jiangsu University, Zhenjiang 212013, China \\ ${ }^{2}$ School of Mechanical Engineering, Jiangsu University, Zhenjiang 212013, China \\ ${ }^{3}$ SUMEC Hardware and Tools Co., Ltd., Nanjing 210032, China
}

\begin{abstract}
Laser dynamic flexible forming (LDFF) is a novel high velocity forming (HVF) technology, in which the foil metal is loaded by laser shock wave. Strain localization is readily to occur around the bulge edge, which will result in the ultimate dynamic failure. In this work, the microstructures before and after dynamic fracture are characterized by transmission electron microscopy (TEM) to investigate the dynamic failure mechanism. The plastic deformation regions of copper foil are composed of shock compression, strain localization and bulge. Microstructure refinement was observed in three different plastic deformation regions, particularly, dynamic recrystallization (DRX) occurs in the strain localization and bulge regions. In bulge region, extremely thin secondary twins in the twin/matrix (T/M) lamellae are formed. The microstructure features in the strain localization region show that superplastic flow of material exists until fracture, which may be due to DRX and subsequent grain boundary sliding (GBS) of the recrystallized grains. The grain coarsening in strain localization region may degrade the material flowing ability which results in the dynamic fracture.
\end{abstract}

Keywords: laser dynamic flexible forming; strain localization; dynamic failure; dynamic recrystallization; grain coarsening

\section{Introduction}

High velocity forming (HVF) can improve the formability of metal sheet in comparison to conventional quasistatic metal sheet forming, so HVF has attracted a great attention [1]. HVF methods at different length scales [2] include electromagnetic forming [3], explosive forming [4] and laser dynamic forming (LDF) [5]. Explosive forming and electromagnetic forming are applicable for macroforming. Laser beam with controllable energy can radiate a very small local region, so LDF can realize plastic forming at micro/nanoscale [6-12]. During micro-forming, the spatial distribution of laser shock wave pressure has a great impact on forming quality. A low density flexible rubber introduced between the laser beam and workpiece can accomodate laser shock wave pressure [13], and so improved micro-forming method is called laser dynamic flexible forming (LDFF) [14]. Under the

*E-mail: lip@ujs.edu.cn action of laser shock wave pressure, the desired micro-feature is obtained by the constraint of flexible pad [15] or rigid micromold. Flexible Pad Laser Shock Forming (FPLSF) is a kind of mold-free micro-forming technique, which can fabricate simple micro-features on metal sheet [15] while micromold based LDF can fabricate complex microfeatures on metal sheet [16]. To improve the forming precision of micro-features in micromold based LDF, Shen et al. [17] adopted the plasticine layer as pressure-carrying medium to reduce the rebound effect.

The material at high strain rates can be superplastically deformed [18]. Many research works have been focused on enhanced formability in electromagnetic forming $[19,20]$, or explosive forming [21]. The enhanced formability may be due to dynamic recrystallization (DRX) and subsequent superplastic flow by grain boundary sliding (GBS) of the recrystallized grains [22]. The enhanced formability in LDF has also attracted great attention. Cheng [23] found that a large dislocation 
density and substructure dominate the microstructure of copper foil, which results in the improved formability of LDF. Liu et al. [24] concluded that DRX is responsible for the high plastic strain of titanium foil under laser shock wave. But the inertia effects, plastic strain and plastic strain rate decrease slightly in FPLSF. Subgrain, high dislocation density and grain refinement region appeared to be a minor component in all of the microstructures of the copper foil as compared with LDF [25]. As the strain rate in LDF induced by laser shock wave can reach $10^{6} \mathrm{~s}^{-1}$ to $10^{7} \mathrm{~s}^{-1}$ [5], during this kind of extreme deformation, strain localization is readily to occur which may result in the ultimate failure. The inhibition of dynamic failure is essentially indispensable for the engineering applications. In order to investigate this kind of dynamic failure phenomena, the formation of dynamic failure has been investigated by numerical simulation [26] and experiments [27] in our previous works. The dynamic fracture modes under different laser energies have been characterized by optical microscopy (OM) and scanning electron microscopy (SEM) observations [27]. In order to better understand the dynamic failure mechanism in LDFF, this paper studies the dynamic failure by transmission electron microscopy (TEM). The microstructures before and after dynamic fracture are characterized.

\section{Laser dynamic flexible forming experiments}

Fig. 1a shows the schematic drawing of the experimental setup of LDFF. To improve laser shock efficiency, the polyurethane rubber (thickness: $100 \mu \mathrm{m}, 200 \mu \mathrm{m}$ and $300 \mu \mathrm{m}$, Shore A hardness: 70) is used as the pressure-carrying medium [14]. A laser pulse (pulse width: $8 \mathrm{~ns}$, wavelength: $1064 \mathrm{~nm}$ ) is focused on the ablative medium (black paint), and then the black paint gets ionized into high pressure plasma. As the plasma is confined by the confining medium ( $\mathrm{K} 9$ glass), plasma-induced shock wave propagates into the rubber which is used to load the $10 \mu \mathrm{m}$ copper foil with a composition of $0.2 \% \mathrm{Sn}, 0.5 \% \mathrm{Zn}$, $0.5 \% \mathrm{~Pb}, 0.5 \% \mathrm{Ni}, 0.5 \% \mathrm{Fe}, 0.2 \% \mathrm{Sb}, 0.5 \%$
$\mathrm{S}, 0.2 \% \mathrm{As}, 0.1 \% \mathrm{Bi}$ and $\mathrm{Cu}+\mathrm{Ag}$ balance (in wt.\%). After laser shock, the micro-bulge feature is formed under the constraint of micromold (diameter: $250 \mu \mathrm{m}$ ), as shown in Fig. 1b. During microforming, strain localization is formed around the bulge edge (the red region in the sample). Such localized deformation will evolve into the dynamic failure as laser energy increases. Fig. 1c shows a few micrometers wide strain localization region.

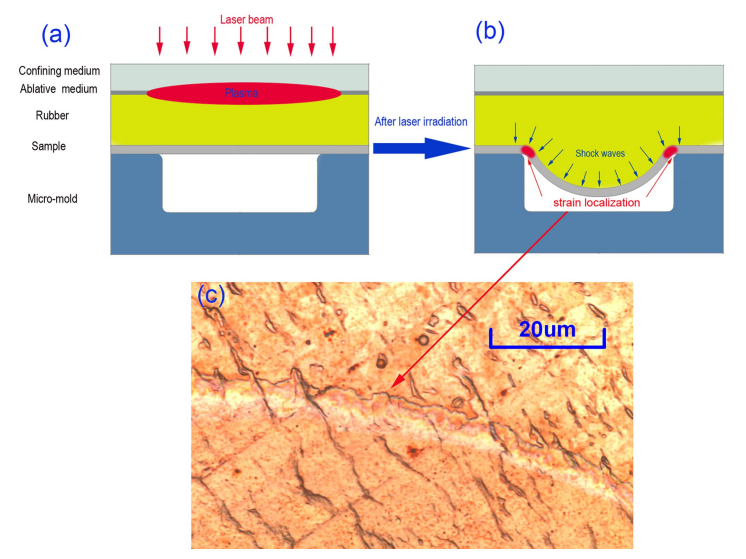

Fig. 1. (a) schematic drawing of the experimental setup of LDFF; (b) the sample after LDFF; (c) microtopography of strain localization phenomenon.

\section{Results and discussion}

\subsection{Optical microscopy (OM) observa- tions}

Zeiss Axio CSM 700 true color confocal microscope is used to measure the surface micro topographies and average surface roughness of the bulge samples. Fig. 2a displays the 3D microtopography of the bulge sample, and the corresponding 2D profile is shown in Fig. 2b. Fig. 2c demonstrates the effect of rubber thickness on forming depth. The experimental results show that the forming depth increases with laser energy for different thicknesses of rubber. When the laser energy remains unchanged, the maximum forming depth decreases with the increase of rubber thickness. This is because the rubber needs more kinetic energy to drive itself to produce elastic deformation so as to load the workpiece when the thickness of rubber increases. 

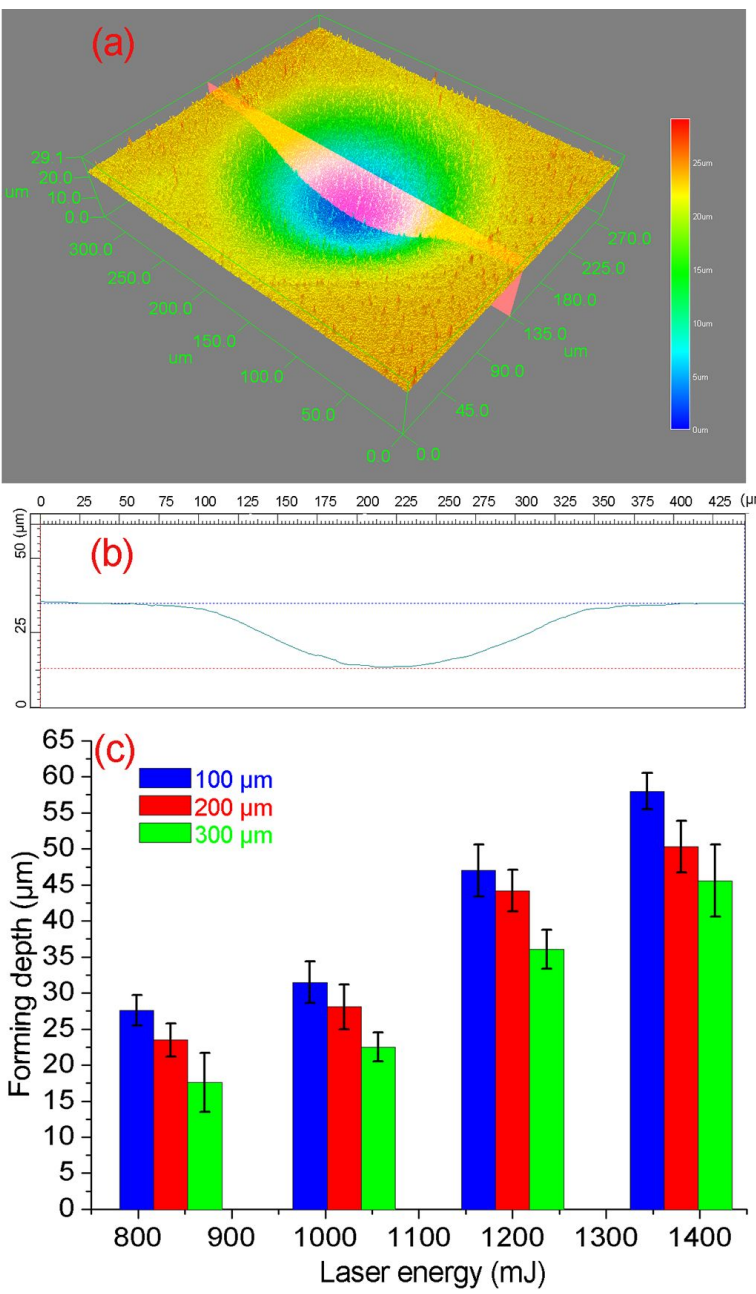

Fig. 2. (a) 3D microtopography of the bulge sample; (b) 2D profile of the bulge sample; (c) the effect of rubber thickness on forming depth.

Fig. 3 summarizes the average surface roughness $(\mathrm{Ra})$ in different regions under $1020 \mathrm{~mJ}$. From Fig. 3a, it can be found that the value of Ra of raw material is $0.268 \mu \mathrm{m}$. After $1020 \mathrm{~mJ}$ laser energy was applied, the value of $\mathrm{Ra}$ in shock compression region reduced to $0.197 \mu \mathrm{m}$, as is shown in Fig. 3b. This is due to the fact that the rubber loading flattens the peak height of the roughness wave. However, the value of $\mathrm{Ra}$ in the bulge region is a little bit higher than that in the shock compression region, as is shown in Fig. 3c. And surface roughening in bulge region is caused by plastic deformation. The experimental results show that good surface quality could be obtained under the rubber loading.

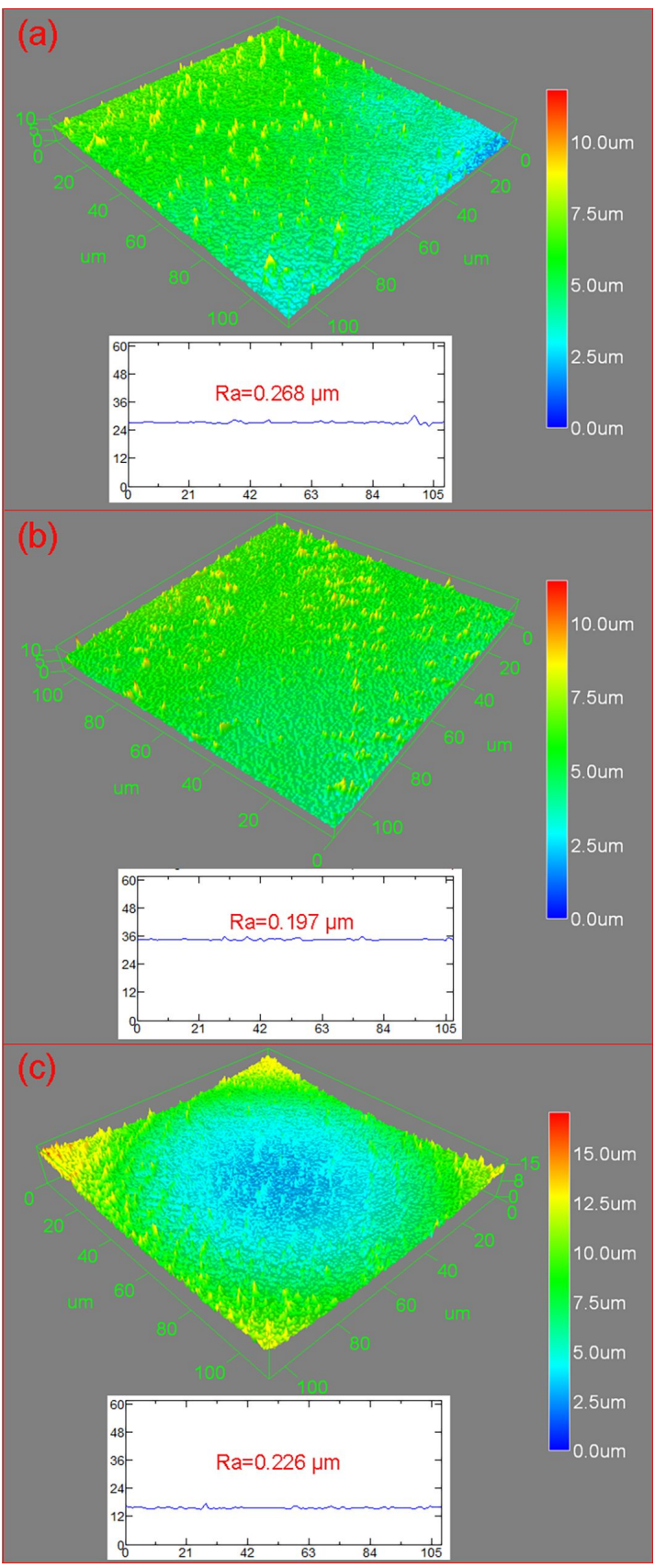

Fig. 3. Average surface roughness in different regions under $1020 \mathrm{~mJ}$ : (a) raw material; (b) shock compression region; (c) bulge region.

\subsection{Transmission electron microscopy (TEM) observations}

Microstructure characteristics of the copper samples at different deformation regions have been studied to uncover the underlying dynamic failure 
mechanism. Two typical bulge samples (with and without failure) are selected for TEM observations, as is shown in Fig. 4. For the bulge sample without failure (Fig. 4a and Fig. 4b), the plastic deformation regions of copper foil are composed of shock compression (region I), strain localization (region II) and bulge (region III), as shown in Fig. 5a. For the bulge sample with failure (Fig. 4c and Fig. 4d), the plastic deformation regions are composed of region I and region II. Fig. 5b presents the schematic illustration of the observed microstructures in different regions, which will be discussed below in more detail.

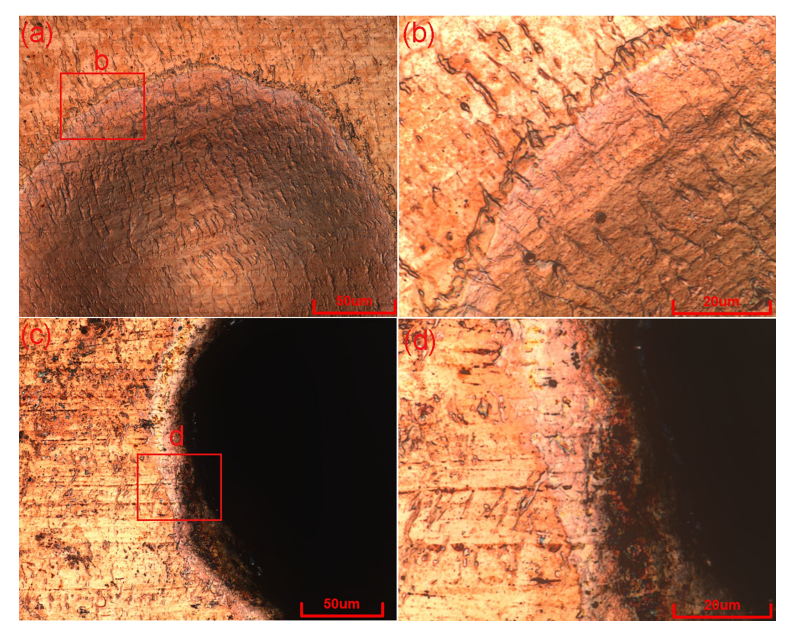

Fig. 4. The bulge samples under different laser energies: (a) and (c), respectively, show the images of sample under $1380 \mathrm{~mJ}$ and $1800 \mathrm{~mJ}$; (b) the higher magnification image of the rectangle region $b$ in (a); (d) the higher magnification image of the rectangle region $\mathrm{d}$ in (c).

\subsubsection{Shock compression region}

In region I, different dislocation characteristics are generated under laser shock compression. Fig. 6a shows a high density of dislocation lines (DLs). When the dislocation density is up to a certain value, dislocation tangles (DTs) and dislocation cells (DCs) are developed to reduce the total free energy of the high-density dislocations [28]. The pile-up of DLs will result in the formation of dislocation walls (DWs) and dense dislocation walls (DDWs) in original grains, so a lot of dislocation cells (DCs) are observed in Fig. 6b. The sizes
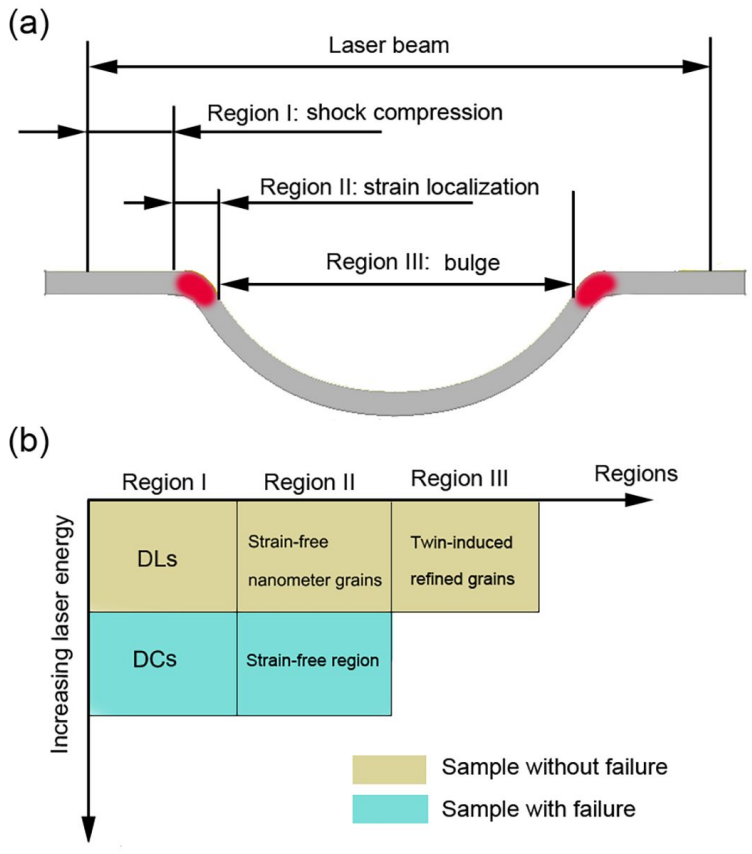

Fig. 5. (a) A schematic illustration of plastic deformation regions of the copper foil; (b) the observed microstructures in different regions.

of DCs range from submicron to micron. The corresponding selected area electron diffraction (SAED) pattern shows no obvious misorientation across the DCs walls. The lack of any obvious misorientation may be due to that the strain in this region is very low. This morphology is similar to that observed in dynamic plastic deformed (DPD) $\mathrm{Cu}$ with very low plastic strain [29]. From the observed results, it can be found that DCs play a significant role in the grain refinement mechanism of laser shock compression of copper foil.

\subsubsection{Strain localization region}

Adjacent to the high-density dislocations region, the strain localization region is observed in Fig. 7a. Obviously this region is completely recrystallized. Several equiaxed nanometer grains are generated in the transition region, as marked by 1-8 in Fig. 7a. The corresponding SAED pattern shows continuous diffraction rings, which indicates that randomly oriented nanometer grains are formed. These microstructure characteristics indicate that DRX occurs in this forming region. These 


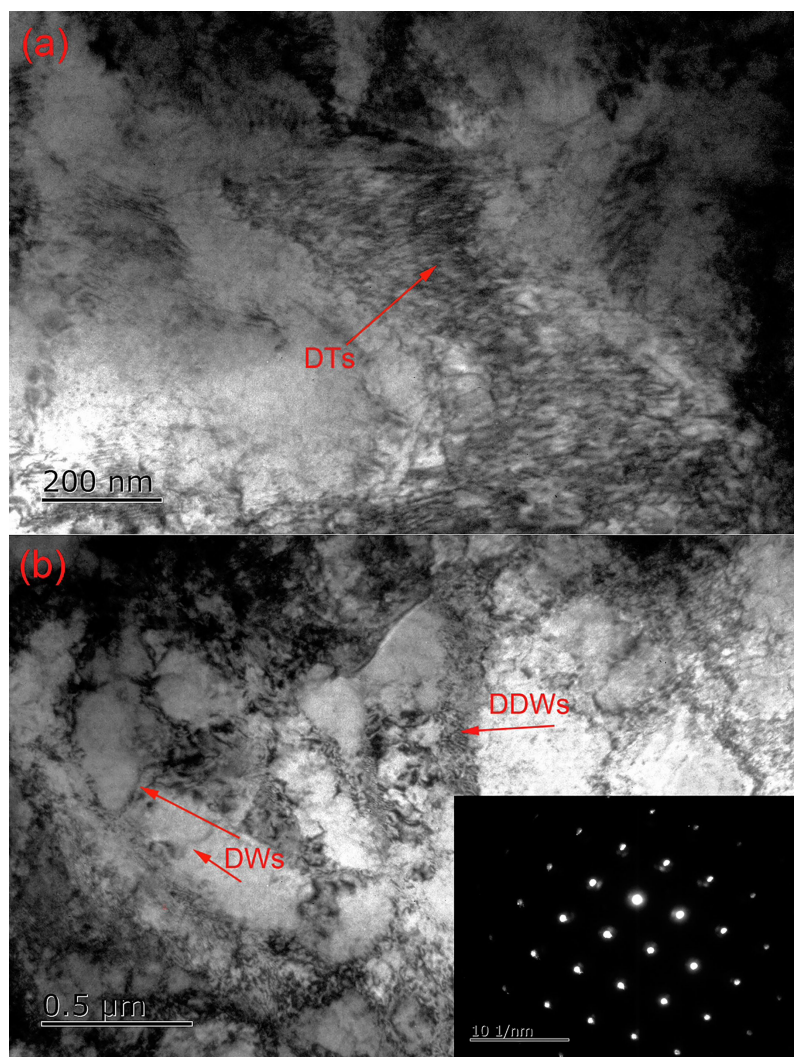

Fig. 6. TEM micrographs of region I under different laser energies: (a) $1380 \mathrm{~mJ}$ and (b) $1800 \mathrm{~mJ}$.

newly formed grains $(\sim 40 \mathrm{~nm})$ are much smaller than those observed in region I. These equiaxed nanometer grains evidently result from DRX. The similar results were also observed in the $\mathrm{Mg}$ alloy [30] and titanium alloy [31] subjected to shear localization. Moiré fringe features were observed in the interfacial region of the dislocations and DRX regions. The parallel lines of Moire fringes are generally caused by the different spacing and orientation of overlapping crystals. Moiré fringe features in the interfacial region confirm the intermixing of the DRX-induced nanometer grains within dislocations matrix. Interestingly, the parallel lines of Moiré fringes show the same orientation, which is probably due to the coherency stress fields. When the material is flowing into the die, it is subjected to a circumferential compressive strain combined with radial tensile strain.

High density of dislocations is observed along the grain boundaries of DRX-induced nanometer
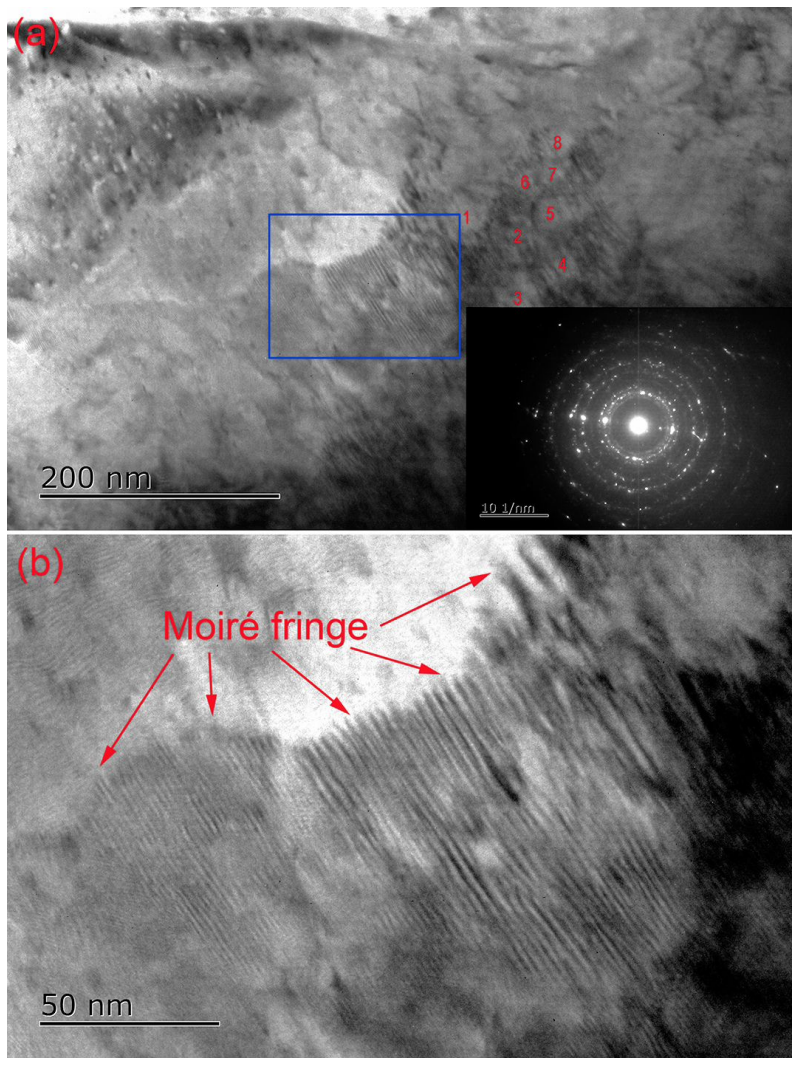

Fig. 7. (a) TEM micrograph of region II under $1380 \mathrm{~mJ}$; (b) the higher magnification image of the Moiré fringe features region.

grains. These dislocation features may be caused by GBS deformation. GBS is believed to be responsible for high plastic strains at the nano-scale. In order to allow for continuous, solid-state flow accommodating high strains and high strain rates, the grain boundary region is temporarily transformed to be amorphous. Thus the DRX grains can easily rotate, slide, and glide past each other to accommodate the superplastic flow [32]. From these TEM observations, it can be inferred that the dislocations, DRX induced equiaxed nanometer grains, and GBS are generated in that sequence during forming.

When the laser energy increases to be $1800 \mathrm{~mJ}$, the plastic deformation is increased in this region. The dynamic recovery (DRV) and grain growth phenomena (marked by $\mathrm{R}$ ) are observed in Fig. 8. These results may be attributed to the high temperature rise in the strain localization region. 
The temperature rise, $\Delta \mathrm{T}$, can be obtained by the following equation:

$$
\beta_{\text {int }} \int_{\varepsilon_{y}}^{\varepsilon_{f}} \delta_{i j} d \varepsilon_{i j}^{p}=\rho C_{p} \Delta T
$$

where $\delta_{\mathrm{ij}}$ denotes the stress, $\epsilon_{\mathrm{ij}}^{\mathrm{p}}$ the plastic strain, the subscripts $y$ and $\mathrm{f}$ stand for yield and failure, $\rho$ is the material density $\left(\rho=8.9 \times 10^{3} \mathrm{~kg} / \mathrm{m}^{3}\right), \mathrm{C}_{\mathrm{p}}$ is the heat capacity $\left(\mathrm{C}_{\mathrm{p}}=394 \mathrm{~J} / \mathrm{kg} \cdot{ }^{\circ} \mathrm{C}\right)$ and $\beta_{\text {int }}$ is the thermo-mechanical conversion ratio $\left(\beta_{\mathrm{int}}=0.9\right)$. According to the above equation, the temperature rise $\Delta \mathrm{T}$ depends on the strain. The high heat pulse might induce DRV or grain coarsening in $\mathrm{Cu}$ [33].

It was also evident that several filaments were formed on the serrated fracture boundaries in Fig. 8. These filaments are parallel to the radius tensile direction, which indicates the high local strain in the fracture region. The serrated fracture boundaries may be caused by the interlinkage of the elongated cavities. The filaments formation is related to the lattice diffusion [34] during GBS. These filament features indicate that the GBS-induced superplastic flow also occurs during fracture process.

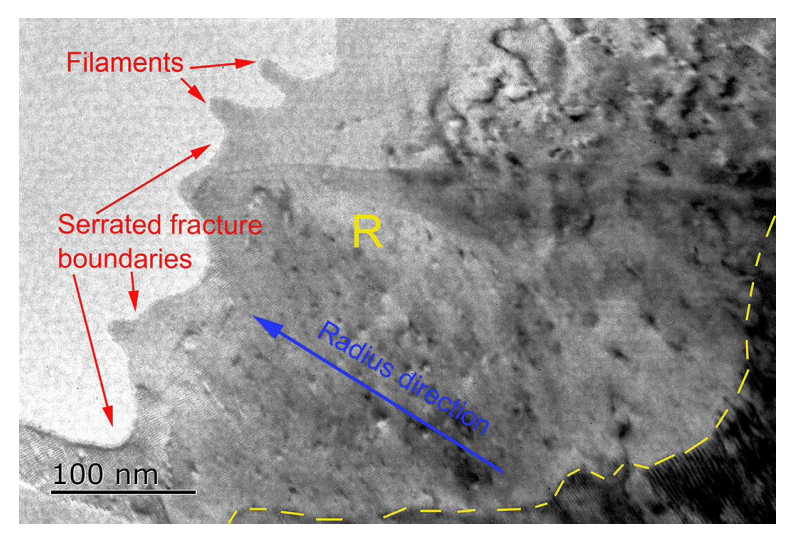

Fig. 8. TEM micrograph of region II under $1800 \mathrm{~mJ}$.

During high temperature deformation, the temperature-induced grain growth makes it difficult for plastic deformation to continue, and the strength of grain boundaries is much lower than that of grain interior. Consequently, superplastic flow is suppressed due to the grain coarsening and intergranular fracture is readily to occur.

\subsubsection{Bulge region}

Fig. 9a shows the typical microstructures in region III. Abundant equiaxed nanometer grains are observed, which can be attributed to the high strain and high strain rate. The corresponding SAED pattern also shows continuous diffraction rings, which indicates that randomly oriented nanometer grains are formed. From Fig. 9b, it can be found that the average grain size is about $20 \mathrm{~nm}$, which is smaller than the recrystallized grain size in region II. Another difference of the recrystallized grains between region II and region III is that dislocations are still observed in region III. These two differences may be due to the occurrence of severe plastic deformation (SPD) in the bulge region. The dislocations boundaries and twin boundaries comprise the boundaries of nanometer grains in bulge region.

Within some nanometer grains, twins can be clearly observed, as indicated by the red circles in Fig. 9b. In each nanometer grain, the thin twin/matrix (T/M) lamellar structure were formed by the parallel multiple twin boundaries. Fig. 9c shows the higher magnification image of the multiple twins inside the nanometer grains. The thickness of the T/M lamellae (as marked by the yellow lines) ranges from several to tens of nanometers. These parallel multiple twins in different nanometer grains are oriented in different directions. And the nanometer grains have a linear distribution, as indicated by the yellow lines in Fig. 9b. According to these two characteristics, it can be inferred that these nanometer grains in bulge region result from the breakdown of the strain-induced elongated grains and the rotations of the broken crystallites. At last, these fragments progressively evolve into the randomly orientated nanometer grains.

Extremely thin secondary twins in the T/M lamellae are formed in nanometer grains, as indicated by the red lines in Fig. 9c. The size of the secondary twins is about a few nanometers. Secondary twins are generated in the T/M lamellae when stressed orientation is favorable. For example, secondary twins in the T/M lamellae phenomena are observed in a $\mathrm{Cu}$ sample processed by surface mechanical grinding treatment (SMGT) [35]. Secondary twins further 
subdivide the T/M lamellar structure into extremely fine prismatic nanosized blocks, as shown in Fig. 9c.

Secondary twins in this case may be due to the fact that the rubber loads the sample again (second loading [26]) during LFDF. So the bulge region is subjected to double dynamic loading. Compared with the T/M lamellar structure, the proportion of secondary twins is comparatively rare. It can be inferred that the rubber's first loading plays a leading role in the microstructure evolution. Rubber's first loading-induced T/M lamellar structure makes great contribution to the grain refinement. Secondary twins further refine the T/M lamellar structure.

\subsubsection{Dynamic failure mechanism}

According to the investigations by Taylor et al. [36], the mechanical energy applied on the sample transforms partly into the thermal energy and the stored energy of cold work [37]. It means that more and more mechanical energy is converted into heat and dynamic stored energy. So the thermal energy and the dynamic stored energy of cold work are two significant factors for the dynamic failure.

In order to understand the formation mechanism of dynamic failure, Zener et al. [38] proposed a competing mechanism based on the thermal energy. At the initial stage of forming, the strain-rate hardening effects dominate over the thermal softening effects. As the material plastic deformation continues, the temperature rise phenomenon becomes more and more apparent, so the thermal softening effects dominate over the strain-rate hardening effects which results in the unstable material flow. The unstable material flow will result in the ultimate failure. And it is generally admitted that the high temperature rise may lead to the formation of DRX [39].

However, Rittel et al. [31, 40] proposed a new perspective on dynamic failure mechanism from the aspect of dynamic stored energy of cold work. Through the experimental investigations, they found that the formation of DRX completely depends on the dynamic stored energy. And the required dynamic mechanical energy is constant.

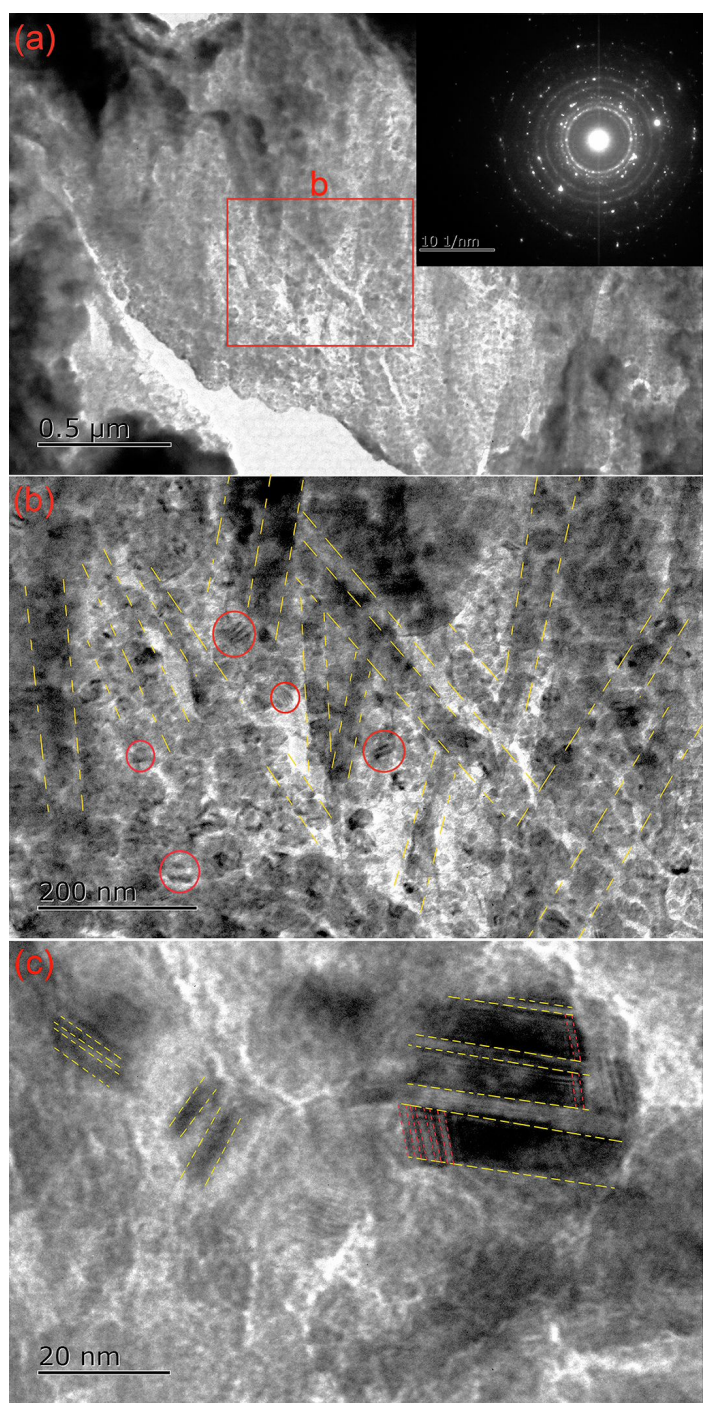

Fig. 9. (a) TEM micrograph of region III under $1380 \mathrm{~mJ}$; (b) the higher magnification image of region $\mathrm{b}$ in $(\mathrm{a})$; (c) the higher magnification image of the T/M lamellar structure and secondary twins.

This constant mechanical energy indicates a state beyond which the microstructure of material can be no more modified, so that the other mechanical energy transforms into heating. Rittel et al. [31] pointed out emphatically that DRX occurs prior to the dynamic failure, and triggers the subsequent dynamic failure.

From TEM observations in region II, DRX also occurs prior to the dynamic failure in this work. Because the required dynamic mechanical energy 


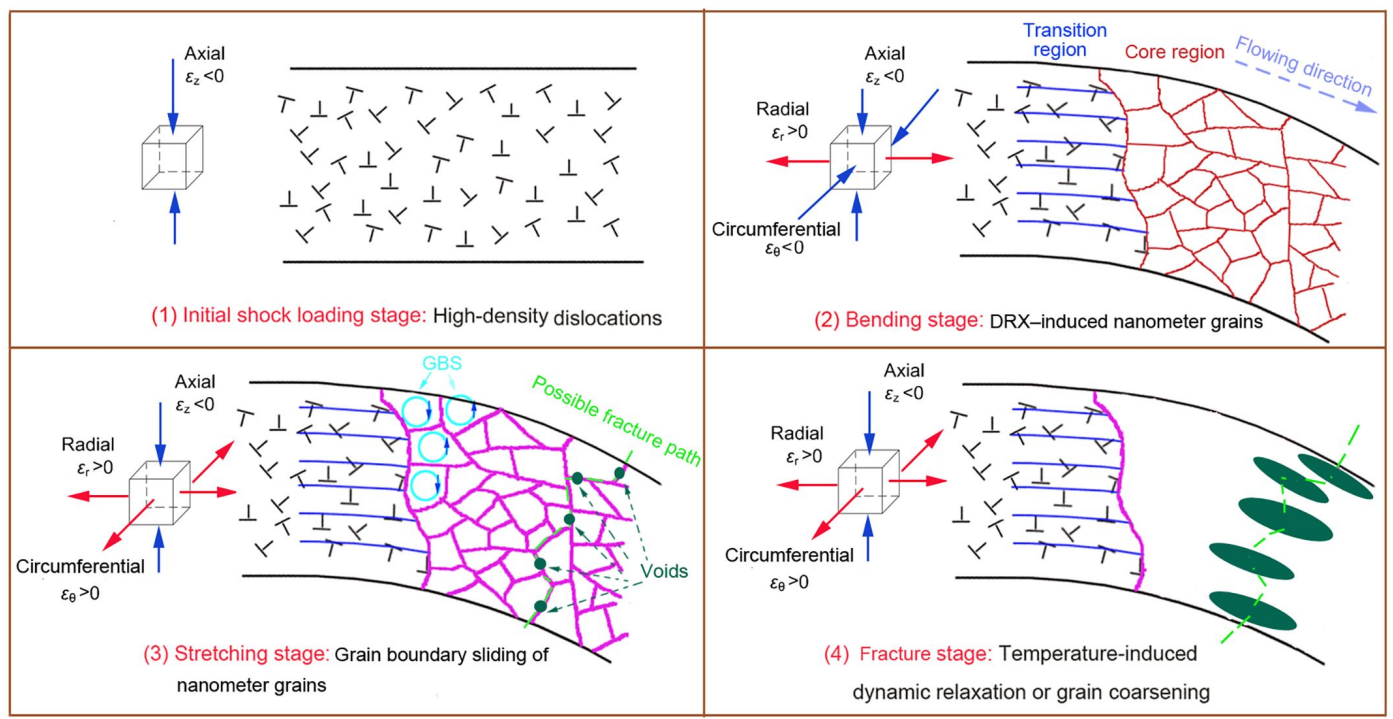

Fig. 10. Schematic illustration showing the possible microstructural evolution process of copper foil in LDFF.

is constant, the other mechanical energy transforms into heating, which leads to the DRV or grain coarsening in region II. The grain coarsening makes the mechanical properties of region II become unstable. So, the grain coarsening after DRX can be regarded as the possible reason for the dynamic failure.

The possible microstructural evolution process is summarized in Fig. 10, including the following four typical states: (1) Initial shock loading stage: high-density dislocations are generated; (2) Bending stage: DRX-induced nanometer grains are formed under shock compression deformation; (3) Stretching stage: in order to accommodate the high strain at the nanoscale, GBS becomes the dominant deformation mechanism. However, the voids easily nucleate under the tension stress; (4) Fracture stage: as the stretching deformation continues, heat-induced grain growth phenomena may degrade the material flowing ability, the local high tensile stress makes the voids grow up and coalesce to form cracks.

\section{Conclusions}

To investigate dynamic failure mechanism of copper foil under LDFF, the microstructures before and after dynamic fracture have been characterized by TEM. Some important conclusions are listed as follows:

1. The forming depth increases with laser energy under different thicknesses of rubber, and the forming depth decreases with the increase of rubber thickness when the laser energy remains unchanged. The good surface quality could be obtained under the rubber loading.

2. Microstructure refinements are observed in three different plastic deformation regions, particularly, dynamic recrystallization (DRX) occurs in the strain localization and bulge regions. The microstructure features in strain localization region show that superplastic flow of material exists until fracture, which may due to DRX and subsequent grain boundary sliding (GBS) of the recrystallized grains.

3. In bulge region, extremely thin secondary twins in the twin/matrix (T/M) lamellae are formed in nanometer grains, which may be due to the occurrence of SPD in this region.

4. DRX occurs prior to the dynamic failure in this work. Because the required dynamic mechanical energy is constant, the other mechanical energy transforms into heating, 
which leads to the DRV or grain coarsening in region II. The grain coarsening makes the mechanical properties of region II become unstable. So the grain coarsening after DRX can be regarded as the possible reasons for the dynamic failure.

\section{Acknowledgements}

This work is supported by the China Postdoctoral Science Foundation (Grant No. 2019M651724) and the National Natural Science Foundation of China (Grants No. 51675243 and 52075226).

\section{References}

[1] DAEHN G.S., High-velocity metal forming: ASM International, Materials Park, Ohio, 2006.

[2] Zhang Y., Babu S. S., Prothe C., Blakely M., Kwasegroch J., Laha M., J. Mater. Process. Technol., 211 (2011), 944.

[3] Khardin M., Harhash M., Chernikov D., Glushchenkov V., Palkowski H., Compos. Struct., 252 (2020), 112729.

[4] Iyama H., Higa Y., Nishi M., Itoh S., Int. J. Mult., 11 (2017), 233.

[5] Li J., Gao H., Cheng G.J., J. Manuf. Sci. Eng.-Trans., ASME 132 (2010), 061005.

[6] Barati Darband G., Aliofkhazraei M., KhorSAND S., Sokhanvar S., Kaboli A., Arab. J. Chem., 13 (2020), 1763.

[7] Li J., Chung T.F., Chen Y.P., Cheng G.J., Nano Lett., 12 (2012), 4577.

[8] Xiong F., Yang H., LiU K., Man J., Chen H., Opt. Laser Technol., 120 (2019), 105762.

[9] Jin S., Wang Y., Motlag M., Gao S., Xu J., Nian Q., Wu W., Cheng G.J., Adv. Mater., 30 (2018).

[10] Choi D.C., Kiм H.S., Opt. Lasers Eng., 124 (2020), 105799.

[11] Jin S., Zhou Z., SAKR E.S.A., Motlag M., HuAng X., Tong L., Bermel P., Ye L., Cheng G.J., Small, 15 (2019), e1900481.

[12] Man J., Yang H., Wang Y., Chen H., Xiong F., Opt. Laser Technol., 119 (2019), 105669.

[13] Shen Z., WANG X., LiU H., WANG Y., WANG C., Appl. Surf. Sci., 327 (2015), 307.

[14] Wang X., Du D., Zhang H., Shen Z., LiU H., Zhou J., Int. J. Mach. Tools Manuf., 67 (2013), 8.

[15] Nagarajan B., Castagne S., Wang Z., Zheng H. Y., NADARAJAN K., Int. J. Mater. Form., 10 (2017), 109.

[16] Yu C., Gao H., Yu H., Jiang H., Cheng G.J., Appl. Phys. Lett., 95 (2009), 091108.

[17] Shen Z., Zhang J., LiU H., Wang X., MA Y., Int. J. Mach. Tools Manuf., 141 (2019), 1.

[18] Balanethiram V.S., Daehn G.S., Scr. Mater., 30 (1994), 515.
[19] Jenab A., Green D.E., Alpas A.T., GolOVASHCHENKo S.F., J. Mater. Process. Technol., 255 (2018), 914.

[20] Psyk V., Risch D., Kinsey B.L., TekKaya A.E., KleINer M., J. Mater. Process. Technol., 211 (2011), 787.

[21] Mynors D.J., Zhang B., J. Mater. Process. Technol., 125 (2002), 1.

[22] Murr L.E., Pizaña C., Metall. Mater. Trans. A-Phys. Metall. Mater. Sci., 38 (2007), 2611.

[23] Cheng G.J., Pirzada D., Ming Z., J. Appl. Phys., 101 (2007), 063108.

[24] LiU H.X., Hu Y., Wang X., Shen Z.B., Li P., Gu C.X., Mater. Sci. Eng. A-Struct. Mater. Prop. Microstruct. Process., 564 (2013), 13.

[25] Nagarajan B., Castagne S., Wang Z., Zheng H. Y., Appl. Phys. A-Mater. Sci. Process., 121 (2015), 695.

[26] Shen Z., Liu H., Wang X., Wang C., Appl. Surf. Sci., 369 (2016), 288.

[27] Shen Z., Zhang J., Li P., LiU H., Yan Z., Ma Y., J. Manuf. Process., 37 (2019), 82.

[28] Wang K., Tao N.R., LiU G., Lu J., Lu K., Acta Mater., 54 (2006), 5281.

[29] LI Y. S., TAO N. R., Lu K., Acta Mater., 56 (2008), 230.

[30] Sun H.Q., Shi Y.N., Zhang M.X., Lu K., Acta Mater., 55 (2007), 975.

[31] Rittel D., Landau P., Venkert A., Phys. Rev. Lett., 101 (2008), 165501.

[32] Esquivel E.V., Murr L.E., Mater. Sci. Eng. A-Struct. Mater. Prop. Microstruct. Process., 409 (2005), 13.

[33] Mishra A., Kad B., Gregori F., Meyers M., Acta Mater., 55 (2007), 13.

[34] CaO F., Li Z., Zhang N., Ding H., Yu F., Zuo L., Mater. Sci. Eng. A-Struct. Mater. Prop. Microstruct. Process., 571 (2013), 167.

[35] TAO N.R., LU K., Scr. Mater., 60 (2009), 1039.

[36] TAYlor G.I., Quinney H., Proc. R. Soc. London Ser. A-Math. Phys. Eng. Sci., A413 (1934), 307.

[37] Rittel D., Wang Z.G., Merzer M., Phys. Rev. Lett., 96 (2006), 075502.

[38] Zener C., Hollomon J. H., J. Appl. Phys., 15 (1944), 22.

[39] Xu Y., Zhang J., Bai Y., Meyers M.A., Metall. Mater. Trans. A-Phys. Metall. Mater. Sci., 39 (2008), 811.

[40] RitTel D., $9^{\text {th }}$ International Conference on Mechanical and Physical Behaviour of Materials under Dynamic Loading, 2009, p. 955. 